\title{
Prevalence of sarcopenia among hospitalized internal medicine patients: A cross-sectional single-center pilot study according to EWGSOP2 criteria
}

\author{
Vrbova P, Smaha J, Stepan J, Tobias D, Kuzma M, Payer J, Koller T \\ 5th Department of Internal Medicine, Comenius University Faculty of Medicine, University Hospital \\ Bratislava Ruzinov, Bratislava, Slovakia. tomas.koller@fmed.uniba.sk
}

\begin{abstract}
INTRODUCTION: Sarcopenia is associated with a higher risk of adverse outcomes among hospitalized patients with chronic diseases. Currently, an unmet need lies in its low diagnostic yield. We conducted a pilot study with the aim to screen hospitalized patients for sarcopenia using the EWGSOP2 criteria.

PATIENTS AND METHODS: A cross-sectional study among hospitalized patients in internal medicine was carried out, inclusion criteria: age $>55$, ability to stand-up from a chair. Exclusion criteria: bed-bound, terminal stages of the disease, ICU stay. We measured hand-grip strength, fat-free mass by bioimpedance and short physical performance battery test (SPPB). Sarcopenia was diagnosed in low hand-grip strength and low muscle mass (EWGSOP2), severe sarcopenia in sarcopenic patients with low physical performance (SPPBT $\leq 8)$.

RESULTS: 40 patients were enrolled, sarcopenia was diagnosed in $8 / 20(40 \%)$ men and 3/20 (15\%) women $(p=0.15)$, severe sarcopenia in $6 / 20$ men $(30 \%)$ and $2 / 20(10 \%)$ women $(p=0.24) .65 \%$ of men and $40 \%$ of women had SPPBT $\leq 8$, and $60 \%$ of men and $55 \%$ of women had gait speed $\leq 0.8 \mathrm{~m} / \mathrm{s}$.

CONCLUSION: Among hospitalized internal medicine patients sarcopenia and severe sarcopenia are common, particularly in males. New diagnostic criteria provide a relatively simple and applicable tool for screening among internal medicine inpatients (Tab. 3, Ref. 25). Text in PDF www.elis.sk.

KEYWORDS: sarcopenia, prevalence, screening, internal medicine, hospitalized patients.
\end{abstract}

\section{Introduction}

Sarcopenia is defined by a progressive and generalized muscle loss(1). It is most often caused by aging, but it also accompanies chronic diseases such as heart failure(2), renal insufficiency(3), obstructive pulmonary disease(4), and liver cirrhosis(5). It is associated with a higher risk of adverse outcomes such as decreased physical performance, immobility, falls, fractures, repeated hospitalizations, loss of independence on activities of daily living, low quality of life or death(6). The number of elderly people at risk of dependence is increasing while putting pressure on the country's health, social and economic systems(7). One of the unfulfilled goals of current patient management is the lack of diagnosis of sarcopenia(8). Identifying these patients would be helpful since patient management could be adapted to prevent further muscle loss. The prevalence of sarcopenia in healthy individuals aged 60 70 has been reported between 5-13\%(9). Among patients older than

5th Department of Internal Medicine, Comenius University Faculty of Medicine, University Hospital Bratislava Ruzinov, Bratislava, Slovakia

Address for correspondence: T. Koller, 5th Department of Internal Medicine, Comenius University Faculty of Medicine, University Hospital Bratislava Ruzinov, Ruzinovska 6, SK-826 06 Bratislava, Slovakia.
70 hospitalized at internal medicine, the prevalence of sarcopenia has been recently reported as high as $42 \%(10)$. With such a high incidence of sarcopenia, it would be appropriate to introduce sarcopenia screening in all hospitalized patients.

Diagnostic criteria for sarcopenia have evolved in recent years from the measurement of muscle mass (EWGSOP) to the recently updated consensus of the European working group for sarcopenia in the elderly people (EWGSOP2)(11). This consensus has provided a simplified approach with an aim to increase the diagnostic yield using muscle strength as a principal criterion for sarcopenia. Therefore, we decided to conduct a pilot study with the aim to screen hospitalized internal medicine patients for sarcopenia using the new consensus criteria.

\section{Aims}

To estimate the prevalence of sarcopenia among hospitalized internal medicine patients. First, by comparing the basic anthropometric parameters between the group of hospitalized patients and healthy controls. Second, to estimate the prevalence of sarcopenia and severe sarcopenia among hospitalized patients using the EWGSOP2 consensus criteria. Third, to compare basic anthropometric parameters and serum markers according to sarcopenia status. 


\section{Patients and methods}

We conducted a cross-sectional study among patients hospitalized at the $5^{\text {th }}$ Department of Internal Medicine Comenius University Faculty of Medicine during the period of 2 weeks. This pilot study is a preliminary report from a larger study that is being conducted in a prospective manner with an aim of finding the most suitable and informative diagnostic tool/parameter for sarcopenia in our particular case-mix of inpatients. Inclusion criteria were an acute hospitalization, age above 55 years, ability to stand-up from a chair and ability to cooperate in measuring the hand-grip strength. We excluded patients that were bed-bound on admission, patients with terminal stages of any disease, with active malignant disease and those hospitalized at the intensive care unit. The control group comprised of the age and sex-matched community-dwelling healthy individuals in whom we assessed anthropometric measures.

In all patients and controls, we assessed the following anthropometric parameters: body mass index (BMI, $\left.\mathrm{kg} / \mathrm{m}^{2}\right)$, mid-arm circumference $(\mathrm{cm})$, tricipital skinfold $(\mathrm{cm})$, subscapular skinfold and the hand-grip strength. Skinfolds were measured using a caliper method (personal body fat tester). Hand-grip strength was assessed using a hand-grip meter (Gima S.p.A. Via Marconi, 1, 20060 Gessate, Italy) by calculating an average value from 3 measurements. Normal hand grip strength values were derived from the EWGSOP2 (men $<27 \mathrm{~kg}$, men $<16 \mathrm{~kg})$. Patients with values below the cut-off were considered as having low muscle strength.

In the patient group, muscle mass was assessed using an electrical bio-impedance device ImpediMed IMP SF7 (ImpediMed Inc. 5900 Pasteur Court, Suite 125, Carlsbad, CA, USA). The measurement was carried out in a supine position, all the electrodes were placed unilaterally on a non-dominant upper limb (yellow electrode - next to the ulna head, a red electrode at the center of the dorsal arm at a distance of $2-5 \mathrm{~cm}$ from the yellow electrode) and lower limb (blue electrode - center between the medial and lateral mal- leoli, black electrode - the center of the foot dorsum at a distance of $2-5 \mathrm{~cm}$ from the blue electrode). From the measured parameters, we assessed the fat-free mass and the fat mass in $\mathrm{kg}$, that was converted into the fat-free mass index (FFMI) and fat mass index (FMI) divided by the square of body height. We adopted normal values for FFMI and FMI from the study among 153 men and 451 women in the geographically and ethnically similar population in Austria(12). Normal values in this study were adjusted according to sex and BMI. Patients with values below the estimated reference range were considered as having a low muscle mass.

Functional status of patients was assessed by the short physical performance battery test(13) (SPPBT) by measuring the following parameters: 1 . Chair-stands (s), the time necessary for 5 chair stands without the help of hands, 2. balance test (s), time the patient maintains the balance having feet together, at the semitandem and tandem position for maximum 10 seconds in each. 3. Gait speed, by measuring the time necessary to walk 5 meters $(\mathrm{m} / \mathrm{s})$. From the measured parameters, we calculated the SPPBT score. Patients having an SBBPT score $\leq 8$ were considered as having a low physical performance.

Sarcopenia was diagnosed according to the criteria of the European working group for sarcopenia in elderly people (EWGSOP2) in patients having low hand-grip strength and a low muscle mass (11). Severe sarcopenia was diagnosed in patients having sarcopenia and low physical performance.

We also assessed basic laboratory parameters (hemoglobin, serum creatinine, CRP, serum vitamin D) for comparison between sarcopenic and non-sarcopenic patients.

The study was conducted in accordance with the proceeding of the declaration of Helsinki. All participants signed an informed consent prior to any procedure. The study protocol was approved by the local ethics committee.

Statistical analysis was carried out using R statistical software version 3.5.1 ( $\mathrm{R}$ foundation for statistical computing, Vi-

Tab. 1. Comparison of parameters between the study group and control group.

\begin{tabular}{|c|c|c|c|c|}
\hline & & Healthy controls & Patients & $n *$ \\
\hline & & Women, $\mathrm{n}(\%)$, median [IQR] $\mathrm{n}=20$ & $\mathrm{n}=20$ & $\mathrm{p}^{*}$ \\
\hline \multirow{2}{*}{ Age category (\%) } & 56 to 70 & $10(50.0)$ & $12(60.0)$ & \multirow{2}{*}{0.751} \\
\hline & 71 to 85 & $10(50.0)$ & $8(40.0)$ & \\
\hline Body mass index $\left(\mathrm{kg} / \mathrm{m}^{2}\right)$ & & $25.73[23.43,27.34]$ & $27.27[25.35,32.09]$ & 0.062 \\
\hline Tricipital skinfold $(\mathrm{cm})$ & & $1.6[1.28,1.92]$ & $2.5[2.0,2.92]$ & $<0.001$ \\
\hline Mid-arm circumference $(\mathrm{cm})$ & & $28.0[27.0,31.0]$ & $31.0[27.5,33.0]$ & 0.149 \\
\hline Hand-grip strength $(\mathrm{kg})$ & & $19.41[17.04,21.25]$ & $16.83[14.58,21.08]$ & 0.387 \\
\hline \multirow[t]{2}{*}{ Hand-grip strength $<16 \mathrm{~kg}$} & & $4(20.0)$ & $8(40.0)$ & 0,173 \\
\hline & & Men, $n(\%)$, median [IQR] $n=20$ & $\mathrm{n}=20$ & \\
\hline \multirow{2}{*}{ Age category (\%) } & 56 to 70 & $10(50.0)$ & $11(55.0)$ & \multirow{2}{*}{1} \\
\hline & 71 to 85 & $10(50.0)$ & $9(45.0)$ & \\
\hline Body mass index $\left(\mathrm{kg} / \mathrm{m}^{2}\right)$ & & $28.1[25.45,29.81]$ & $27.4[26.0,29.04]$ & 0.957 \\
\hline Tricipital skinfold $(\mathrm{cm})$ & & $1.75[1.50,1.92]$ & $1.40[1.08,2.05]$ & 0.261 \\
\hline Mid-arm circumference $(\mathrm{cm})$ & & $32.0[28.75,34.25]$ & $30.0[28.0,31.25]$ & 0.128 \\
\hline Hand-grip strength $(\mathrm{kg})$ & & $31.9[24.58,37.67]$ & $25.5[19.1,29.83]$ & 0.024 \\
\hline Hand-grip strength $<27 \mathrm{~kg}$ & & $6(30.0)$ & $12(60.0)$ & 0.06 \\
\hline
\end{tabular}

*difference between patients and healthy controls, chi-square or Fisher test, or Mann-Whitney test 
enna Austria) using an EZR Plugin (Kanda et al. (14)) Results of numerical variables are displayed as medians and interquartile ranges, proportions are displayed as numbers and percentages. Mann-Whitney test was used for comparison of numerical variables and the chi-square test or Fisher test was used to compare proportions. Significant differences were stated in cases when the probability of null-hypothesis was inferior to $5 \%(\mathrm{p}<0.05)$.

\section{Results}

We enrolled 40 patients, 20 men and 20 women, and 40 healthy controls, 20 men and 20 women.
Sex-specific comparison for anthropometric parameters between the patient group and the control group are displayed in Table 1. In women, patients had significantly higher tricipital skinfold compared to healthy controls (2.5 IQR2.02.92 vs. 1.6 IQR1.28-1.92, $\mathrm{p}<0.001)$ and a trend towards higher BMI (27.27 IQR 25.35-32.1 vs. 25.73 IQR23.43$27.34, \mathrm{p}=0.062$ ), but there was no difference in the hand-grip strength (16.83 IQR14.58-21.08 vs. 19.41IQR 17.04-21.25, $\mathrm{p}=0.39$ ) or the prevalence of HGS below $16 \mathrm{~kg}$. In men, patients had comparable BMI and the tricipital skinfold but had lower hand-grip strength and a higher prevalence of hand-grip strength below $27 \mathrm{~kg}$ compared to healthy controls

Tab. 2. Summary statistics and characteristics of the patient group.

\begin{tabular}{|c|c|c|c|c|}
\hline & & Men, $\mathrm{n}=20$ & Women, $\mathrm{n}=20$ & \multirow{2}{*}{$\mathrm{P} *$} \\
\hline & & Median [IQR], n (\%) & Median [IQR], n (\%) & \\
\hline Age & & $70.00[60.75,74.75]$ & $69.50[65.00,73.00]$ & 0.90 \\
\hline $\mathrm{BMI}\left(\mathrm{kg} / \mathrm{m}^{2}\right)$ & & $27.38[26.00,29.04]$ & $27.27[25.35,32.09]$ & 0.93 \\
\hline \multicolumn{5}{|l|}{ Principal diagnosis } \\
\hline Dg. Anemia & & $2(10.0)$ & $3(15.0)$ & \multirow{6}{*}{0.86} \\
\hline Dg. Gastrointestinal disease & & $2(10.0)$ & $4(20.0)$ & \\
\hline Dg. Renal disease & & $1(5.0)$ & $1(5.0)$ & \\
\hline Dg. Cardiovascular disease & & $9(45.0)$ & $7(35.0)$ & \\
\hline Dg. Metabolic disease & & $3(15.0)$ & $4(20.0)$ & \\
\hline Dg. Sepsis & & $3(15.0)$ & $1(5.0)$ & \\
\hline Waist circumference $(\mathrm{cm})$ & & $106.50[101.75,113.25]$ & $100.50[91.75,114.50]$ & 0.33 \\
\hline Mid-arm circumference $(\mathrm{cm})$ & & $30.00[28.00,31.25]$ & $31.00[27.50,33.00]$ & 0.56 \\
\hline Subscapular skinfold $(\mathrm{cm})$ & & $2.00[1.87,2.23]$ & $2.05[1.80,2.70]$ & 0.61 \\
\hline Triceps skinfold $(\mathrm{cm})$ & & $1.40[1.08,2.05]$ & $2.50[2.00,2.92]$ & $<0.01$ \\
\hline Hand-grip strength (kg) & & $25.50[19.08,29.83]$ & $16.83[14.58,21.08]$ & 0.01 \\
\hline \multirow{2}{*}{$\begin{array}{l}\text { Low hand-grip strength } \\
(\text { men }<27, \text { women }<16 \mathrm{~kg})\end{array}$} & no & $8(40.0)$ & $12(60.0)$ & \multirow{2}{*}{0.34} \\
\hline & yes & $12(60.0)$ & $8(40.0)$ & \\
\hline Balance test (s) & & $30.00[23.50,30.00]$ & $30.00[27.25,30.00]$ & 0.48 \\
\hline Gait-speed (m/s) & & $0.60[0.42,1.05]$ & $0.71[0.52,1.10]$ & 0.24 \\
\hline \multirow{2}{*}{ Low gait speed $(\leq 0.8 \mathrm{~m} / \mathrm{s})$} & no & $8(40.0)$ & $9(45.0)$ & \multirow{2}{*}{1.00} \\
\hline & yes & $12(60.0)$ & $11(55.0)$ & \\
\hline Five chair stands (s) & & $14.55[11.00,23.10]$ & $13.25[11.95,15.57]$ & 0.56 \\
\hline \multirow{2}{*}{ Chairstands $>15 \mathrm{~s}$} & no & $12(60.0)$ & $14(70.0)$ & \multirow[t]{2}{*}{0.74} \\
\hline & yes & $8(40.0)$ & $6(30.0)$ & \\
\hline SPPBT $f$ score & & $6.00[5.75,11.00]$ & $9.50[6.75,10.25]$ & 0.41 \\
\hline \multirow{2}{*}{ SPPBT score $\leq 8$} & no & $7(35.0)$ & $12(60.0)$ & \multirow{2}{*}{0.21} \\
\hline & yes & $13(65.0)$ & $8(40.0)$ & \\
\hline Fat-free mass index $\left(\mathrm{kg} / \mathrm{m}^{2}\right)$ & & $20.59[19.03,22.35]$ & $17.76[16.18,20.89]$ & 0.04 \\
\hline \multirow{2}{*}{ Fat-free mas index $<$ normal } & no & $8(40.0)$ & $14(70.0)$ & \multirow{2}{*}{0.11} \\
\hline & yes & $12(60.0)$ & $6(30.0)$ & \\
\hline Fat mass index $\left(\mathrm{kg} / \mathrm{m}^{2}\right)$ & & $7.06[4.98,10.17]$ & $9.87[6.79,11.49]$ & 0.22 \\
\hline \multirow{2}{*}{ Fat mass index $<$ normal } & no & $19(95.0)$ & $15(75.0)$ & \multirow{2}{*}{0.18} \\
\hline & yes & $1(5.0)$ & $5(25.0)$ & \\
\hline Serum kreatinin (umol/l) & & $90.70[76.77,124.25]$ & $73.35[60.95,110.10]$ & 0.13 \\
\hline Hemoglobin (g/l) & & $122.50[110.3,131.3]$ & $120.00[110.0,128.3]$ & 0.77 \\
\hline C-reactive protein $(\mathrm{mg} / \mathrm{l})$ & & $6.15[1.15-47.56]$ & $4.43[1.82,26.97]$ & 0.86 \\
\hline Serum vitamin D (ug/l) & & $16.95[12.15,22.00]$ & $20.05[14.73,27.00]$ & 0.32 \\
\hline \multirow{2}{*}{ Sarcopenia } & no & $12(60.0)$ & $17(85.0)$ & \multirow{2}{*}{0.16} \\
\hline & yes & $8(40.0)$ & $3(15.0)$ & \\
\hline \multirow{2}{*}{ Severe sarcopenia } & no & $14(70.0)$ & $18(90.0)$ & \multirow{2}{*}{0.24} \\
\hline & yes & $6(30.0)$ & $2(10.0)$ & \\
\hline
\end{tabular}

* p value for the sex difference, chi-square or Fisher test or Mann-Whitney test

$f$ SPPBT, short performance physical battery test 
Tab. 3. Comparison of patients' characteristics according to the presence of sarcopenia.

\begin{tabular}{|c|c|c|c|}
\hline & No sarcopenia, $\mathrm{n}=29$ & Sarcopenia, $\mathrm{n}=11$ & \multirow{2}{*}{$\mathrm{P}^{f}$} \\
\hline & Median [IQR], n (\%) & Median [IQR], n (\%) & \\
\hline Age & $69.00[64.00,73.00]$ & $71.00[64.00,75.50]$ & 0.504 \\
\hline Age category (56-70) & $18(62.1)$ & $5(45.5)$ & \multirow{2}{*}{0.477} \\
\hline Age category (71-85) & $11(37.9)$ & $6(54.5)$ & \\
\hline Men & $12(41.4)$ & $8(72.7)$ & \multirow{2}{*}{0.155} \\
\hline Women & $17(58.6)$ & $3(27.3)$ & \\
\hline Height $(\mathrm{cm})$ & $170.00[164.00,178.00]$ & $166.00[165.00,172.50]$ & 0.585 \\
\hline Weight $(\mathrm{kg})$ & $83.00[74.00,95.00]$ & $74.00[66.00,79.00]$ & 0.108 \\
\hline BMI $\left(\mathrm{kg} / \mathrm{m}^{2}\right)$ & $27.77[25.62,31.84]$ & $26.90[24.58,28.78]$ & 0.303 \\
\hline BMI category (18-24.9) & $8(27.6)$ & $3(27.3)$ & \multirow{3}{*}{0.9} \\
\hline BMI category 25-29.9) & $13(44.8)$ & $6(54.5)$ & \\
\hline BMI category $>30$ & $8(27.6)$ & $2(18.2)$ & \\
\hline \multicolumn{4}{|l|}{ Principal diagnosis } \\
\hline Dg. Anemia & $4(13.8)$ & $1(9.1)$ & \multirow{6}{*}{0.932} \\
\hline Dg. Gastrointestinal disease & $5(17.2)$ & $1(9.1)$ & \\
\hline Dg. Renal disease & $2(6.9)$ & $0(0.0)$ & \\
\hline Dg. Cardiovascular disease & $11(37.9)$ & $5(45.5)$ & \\
\hline Dg. Metabolic disease & $4(13.8)$ & $3(27.3)$ & \\
\hline Dg. Sepsis & $3(10.3)$ & $1(9.1)$ & \\
\hline Waist circumference $(\mathrm{cm})$ & $108.00[99.00,115.00]$ & $106.00[98.00,110.00]$ & 0.534 \\
\hline Mid-arm circumference $(\mathrm{cm})$ & $31.00[29.00,33.00]$ & $29.00[26.50,30.50]$ & 0.038 \\
\hline Subscapular skinfold (cm) & $2.10[1.80,2.50]$ & $2.00[1.85,2.30]$ & 0.419 \\
\hline Triceps skinfold $(\mathrm{cm})$ & $2.30[1.50,2.60]$ & $1.50[1.15,2.35]$ & 0.288 \\
\hline Balance test (s) & $30.00[25.00,30.00]$ & $30.00[23.00,30.00]$ & 0.538 \\
\hline Gait-speed (m/s) & $0.71[0.50,1.00]$ & $0.50[0.33,1.22]$ & 0.275 \\
\hline Five chair stands (s) & $13.00[11.00,15.50]$ & $18.00[12.75,24.52]$ & 0.066 \\
\hline Fat mass index $\left(\mathrm{kg} / \mathrm{m}^{2}\right)$ & $8.50[6.11,10.11]$ & $9.53[6.48,11.21]$ & 0,617 \\
\hline Serum bilirubin (umol/l) & $10.90[9.17,15.00]$ & $12.00[9.65,19.59]$ & 0.535 \\
\hline Serum kreatinin (umol/l) & $85.10[70.80,114.00]$ & $87.10[66.45,149.90]$ & 0.515 \\
\hline C-reactive protein $(\mathrm{mg} / \mathrm{l})$ & $6.3[1.97,29.47]$ & $3.4[2.02,22.31]$ & 0.75 \\
\hline Serum urea $(\mathrm{mmol} / \mathrm{l})$ & $5.84[4.73,8.68]$ & $5.10[4.01,9.63]$ & 0.94 \\
\hline Hemoglobin $(\mathrm{g} / \mathrm{l})$ & $120.00[107.00,128.00]$ & $123.00[116.50,137.00]$ & 0.296 \\
\hline Serum vitamin D (ug/l) & $18.40[14.10,25.20]$ & $18.00[14.50,22.10]$ & 0.976 \\
\hline
\end{tabular}

${ }^{f}$ chi-square or Fisher test, or Mann-Whitney test

(25.5 IQR19.1-29.83 vs. 31.9 IQR24.58-37.67, $\mathrm{p}=0.024$ and $60 \%$ vs. $30 \%, \mathrm{p}=0,06)$.

Among 40 hospitalized patients, the median age in both sexes was 70.0 vs. 69.5 respectively. Study group characteristics and the comparison between men and women are displayed in Table 2. Median BMI was in the range of overweight $\left(27.38 \mathrm{~kg} / \mathrm{m}^{2}\right.$ and 27.27 , $\mathrm{p}=0.93$ ). Women had significantly higher tricipital skinfold compared to men (2.5 cm IQR2.0-2.92 vs. 1.4 IQR1.08-2.05), lower hand-grip strength (16.83 kg IQR14.58-21.08 vs. 25.5 IQR19.08$29.83, \mathrm{p}=0.01)$ and lower fat-free mass index (17.76 IQR16.1820.89 vs. 20.59 IQR $19.03-22.35$, $\mathrm{p}=0.04)$. Among the parameters of functional status (chair stands, gait speed, balance) we did not observe any difference between the sexes.

Sarcopenia was diagnosed in $8 / 20(40 \%)$ men and $3 / 20(15 \%)$ women $(p=0.15)$. Severe sarcopenia was diagnosed in $6 / 20$ men $(30 \%)$ and $2 / 20(10 \%)$ women $(p=0.24)$. According to the SPPB test, $65 \%$ of men and $40 \%$ of women had low performance status. According to the gait speed, $60 \%$ of men and $55 \%$ of women had low gait speed. The differences were not statistically significant.
Comparison of selected parameters, with the exception of those that defined sarcopenia, between the group of patients with and without sarcopenia is displayed in Table 3 . We observed no difference in the serum parameters between the groups. Serum vitamin D concentrations were not significantly different between patients with or without sarcopenia, between patients with low or normal hand grip strength, gait speed or SPPB score. Sarcopenic patients had lower mid-arm circumference (29.0 IQR26.5-30.5 vs. 31.0 IQR29.0-33.0, $\mathrm{p}=0.038)$ and a trend towards higher time for chairstands (18.0 IQR12.75-24.52 vs. 13.0 IQR11.0-15.5, $\mathrm{p}=0.06$ ).

\section{Discussion}

In our study, we aimed at sarcopenia screening among hospitalized internal medicine patients. Hospitalized women compared to healthy controls had significantly higher tricipital skinfold while hospitalized men had significantly lower hand grip strength. Among 40 hospitalized patients using the EWGSOP2 criteria, sarcopenia was diagnosed in $40 \%$ of men and $15 \%$ of women, severe 
sarcopenia in $30 \%$ of men and $10 \%$ of women. Patients with or without sarcopenia did not differ in basic laboratory parameters including vitamin $\mathrm{D}$.

This pilot study is an inaugural report with an obvious limitation allowing only for cross-sectional analysis. Clinical significance of our findings and their impact on the outcome of patients will be tested in a prospective manner and by including a higher number of patients. However, this pilot study confirms that the new consensus criteria for diagnosing sarcopenia are feasible in our clinical practice. Hand-grip strength measurement is an easy screening tool that is objective and requires little time for evaluation. Furthermore, bioimpedance techniques for evaluating the muscle mass could be performed bed-side with little dedicated time. These two tests appear as the simplest choice since other alternatives proposed by the current consensus (DXA scan, or CT/ MRI scans) are more time and device demanding. For confirming severe sarcopenia, physical performance tests are more time-consuming. We decided to use a more complex SPPBT, but the current consensus also proposes using the gait speed. We also tested gait speed, and the difference in the diagnostic yield for severe sarcopenia would only be in one male patient decreasing its prevalence from 20 to $17.5 \%$. In clinical practice, there is an advantage in physical testing because it could be repeatedly performed during hospitalization for evaluating early changes in physical status. Some prospective studies have reported, that in a particular group of patients, not all physical performance parameters have an equal impact on the outcome. For example, a study among very old men from Sweden, hand-grip strength and gait speed were associated with independent aging, but not muscle mass or diagnosed sarcopenia(15). Only the prospective arm of our study will find the most informative parameter(s) in the prediction of the adverse outcome among our particular case-mix of inpatients.

The prevalence of sarcopenia and severe sarcopenia in $40 \%$ or $30 \%$ of men and $15 \%$ and $10 \%$ of women was high and suggests that sarcopenia should be screened in patients hospitalized at internal medicine. Some previous reports have found similar prevalence among hospitalized medical patients (16). Among hospitazlied geriatric patients, the prevalence of sarcopenia ranged from $11.4 \%$ (17) up to $51 \%$ and $64 \%$ among acute geriatric patients or elderly patients after myocardial infarction $(18,19)$. However, the previous studies have used different diagnostic criteria not allowing for a direct comparison with our study using EWGSOP2 criteria. One study has compared the prevalence of sarcopenia using both EWGSOP1 and 2 algorithms showing a slightly lower prevalence of 27.7 vs.18.1\% when using EWGSOP2 criteria (20).

The presence of sarcopenia among hospitalized patients increases the risk of adverse outcome. During 2 years after an acute hospitalization, the risk of death has been reported as 4 to 9 times higher $(17,18)$. A systematic review has also reported that sarcopenia increases the risk of hospital related outcomes in elderly people by more than $40 \%$ (21). Therefore, due to the risk of adverse outcome during hospitalization, physicians have an opportunity for applying early measures for preventing further loss of muscle mass and function. Currently, early nutritional intervention with increased protein consumption, supplementation of vitamin D as well as regular physiotherapy have been recommended by the recent guidelines $(22,23)$. Finally, we did not find any significant difference in the vitamin D levels according to sarcopenia status, low hand-grip strength, low gait speed or low physical performance, but this pilot study was not powered for detecting a difference in laboratory parameters.

In our study, the prevalence of sarcopenia and severe sarcopenia was higher in men than in women. The difference was not statistically significant but this was likely due to low numbers $(\mathrm{p}=0.15)$. The prevalence of low functional status was also numerically higher in men (65\%) than in women $(40 \%)$, but low gait speed was found among similar proportions of patients $(60 \%$ vs. $55 \%$ ). Some sex-related differences in the prevalence of sarcopenia and its outcome have been reported $(19,20,24)$. It appears that in men, chronic diseases lead to a more dynamic decline in muscle mass. In contrast, due to different fat distribution, hospitalized women had significantly higher tricipital skin-fold and also higher BMI compared to healthy controls, probably reflecting the greater burden of the metabolic syndrome and its complications on women. This finding also points to an important sex-related difference in body composition and changes in body composition in chronic disease. One recent study has shown that in women with cirrhosis, it was the subcutaneous fat loss and not the muscle mass that was associated with the adverse outcome (25). Therefore, the prognostic value of sarcopenia and functional status should always be separately reported for each sex.

\section{Conclusion}

Among hospitalized internal medicine patients by applying the updated EWGSOP2 criteria we diagnosed sarcopenia and severe sarcopenia in $40 \%$ and $30 \%$ of men and $15 \%$ and $10 \%$ of women. The new diagnostic criteria were confirmed as easily applicable for inpatients. Observed high prevalence of sarcopenia justifies its screening among all internal medicine inpatients allowing for early preventive or therapeutic interventions.

\section{References}

1. Cruz-Jentoft AJ, Baeyens JP, Bauer JM, Boirie Y, Cederholm T, Landi F et al. Sarcopenia: European consensus on definition and diagnosis: Report of the European Working Group on Sarcopenia in Older People. Age Ageing 2010; 39: 412-423.

2. Sanchez-Rodriguez D, Hope S, Piotrowicz K, Benoit F, Czesak J, Dallmeier D et al. Sarcopenia in Acute Care Patients: Protocol for the European Collaboration of Geriatric Surveys: Sarcopenia 9+ EAMA Project. J Am Med Dir Assoc 2019; June 20, epub.

3. Souza VA de, Oliveira D, Barbosa SR, Correa JO do A, Colugnati FAB, Mansur HN et al. Sarcopenia in patients with chronic kidney disease not yet on dialysis: Analysis of the prevalence and associated factors. PLoS One 2017; 12: e0176230.

4. Kim SH, Shin MJ, Shin YB, Kim KU. Sarcopenia Associated with Chronic Obstructive Pulmonary Disease. J Bone Metab 2019; 26: $65-74$. 


\section{$717-722$}

5. Montano-Loza AJ, Meza-Junco J, Prado CMM, Lieffers JR, Bara$\cos$ VE, Bain VG et al. Muscle wasting is associated with mortality in patients with cirrhosis. Clin. Gastroenterol Hepatol 2012; 10: 166-173, 173.e1.

6. Qiu J, Thapaliya S, Runkana A, Yang Y, Tsien C, Mohan ML et al. Hyperammonemia in cirrhosis induces transcriptional regulation of myostatin by an NF-kappaB-mediated mechanism. Proc Natl Acad Sci USA 2013; 110: 18162-18167.

7. World Health Organization. WHO Clinical Consortium on Healthy Ageing. In: Topic focus: frailty and intrinsic capacity 2017. p. 36.

8. Van Ancum JM, Meskers CGM, Reijnierse EM, Yeung SSY, Jonkman NH, Trappenburg MC et al. Lack of Knowledge Contrasts the Willingness to Counteract Sarcopenia Among Community-Dwelling Adults. J. Aging Health 2019; June 3, epub.

9. Shafiee G, Keshtkar A, Soltani A, Ahadi Z, Larijani B, Heshmat R. Prevalence of sarcopenia in the world: a systematic review and metaanalysis of general population studies. J Diabet Metab Disord 2017; 16: 21.

10. Gingrich A, Volkert D, Kiesswetter E, Thomanek M, Bach S, Sieber CC et al. Prevalence and overlap of sarcopenia, frailty, cachexia and malnutrition in older medical inpatients. BMC Geriatr 2019; 19: 120.

11. Cruz-Jentoft AJ, Bahat G, Bauer J, Boirie Y, Bruyere O, Cederholm T et al. Sarcopenia: revised European consensus on definition and diagnosis. Age Ageing 2019; 48: 16-31.

12. Bahadori B, Uitz E, Tonninger-Bahadori K, Pestemer-Lach I, Trummer M, Thonhofer $\mathbf{R}$ et al. Body composition: the fat-free mass index (FFMI) and the body fat mass index (BFMI) distribution among the adult Austrian population - results of a cross-sectional pilot study. Int J Body Compos Res 2006; 4: 123-128.

13. Treacy D, Hassett L. The Short Physical Performance Battery. J Physiother 2018; 64: 61 .

14. Kanda Y. Investigation of the freely available easy-to-use software 'EZR' for medical statistics. Bone Marrow Transplant 2012; 48: 452.

15. Franzon K, Zethelius B, Cederholm T, Kilander L. The impact of muscle function, muscle mass and sarcopenia on independent ageing in very old Swedish men. BMC Geriatr 2019; 19: 153.
16. Gariballa S, Alessa A. Sarcopenia: prevalence and prognostic significance in hospitalized patients. Clin. Nutr. 2013;32:772-776.

17. Atmis V, Yalcin A, Silay K, Ulutas S, Bahsi R, Turgut T et al. The relationship between all-cause mortality sarcopenia and sarcopenic obesity among hospitalized older people. Aging Clin Exp Res 2019. Available from: http://dx.doi.org/10.1007/s40520-019-01277-5.

18. Sipers WMWH, de Blois W, Schols JMGA, van Loon LJC, Verdijk LB. Sarcopenia Is Related to Mortality in the Acutely Hospitalized Geriatric Patient. J. Nutr. Health Aging 2019; 23: 128-137.

19. Santana N de M, Mendes RML, Silva NF da, Pinho CPS. Sarcopenia and sarcopenic obesity as prognostic predictors in hospitalized elderly patients with acute myocardial infarction. Einstein (Sao Paulo) 2019; 17: eAO4632.

20. Reiss J, Iglseder B, Alzner R, Mayr-Pirker B, Pirich C, Kassmann $\mathbf{H}$ et al. Consequences of applying the new EWGSOP2 guideline instead of the former EWGSOP guideline for sarcopenia case finding in older patients. Age Ageing 2019; 48: 719-724.

21. Reijnierse EM, Buljan A, Tuttle CSL, van Ancum J, Verlaan S, Meskers CGM et al. Prevalence of sarcopenia in inpatients 70 years and older using different diagnostic criteria. Nurs Open 2019; 6: 377-383.

22. Gariballa S, Alessa A. Sarcopenia: prevalence and prognostic significance in hospitalized patients. Clin Nutr 2013; 32: 772-776.

23. Dent E, Morley J, Cruz-Jentoft A, Arai H, Kritchevsky S, Guralnik $\mathbf{J}$ et al. International Clinical Practice Guidelines for Sarcopenia (ICFSR): Screening, Diagnosis and Management. J Nutr Heal Aging 2018; 22: 1148-1161.

24. Zadak Z. Prevention and therapy of sarcopenia in the ageing. Vnitr Lek 2016; 62: 671-677.

25. Ebadi M, Tandon P, Moctezuma-Velazquez C, Ghosh S, Baracos VE, Mazurak VC et al. Low subcutaneous adiposity associates with higher mortality in female patients with cirrhosis. J Hepatol 2018; 69: 608-616.

Received July 1, 2019. Accepted July 15, 2019. 